\title{
Fast Wavelet-based Pansharpening of Multi-Spectral Images
}

\author{
Nikolaos Mitianoudis \\ School of Science and Technology \\ International Hellenic University \\ 57001 Thermi - Thessaloniki, Greece \\ Email: n.mitianoudis@ihu.edu.gr
}

\author{
Georgios Tzimiropoulos and Tania Stathaki \\ Communication and Signal Processing Group \\ Imperial College London \\ Exhibition Road \\ SW7 2AZ London, UK \\ Email: g.tzimiropoulos@imperial.ac.uk
}

\begin{abstract}
Remote Sensing systems enhance the spatial quality of low-resolution Multi-Spectral (MS) images using information from Pan-chromatic (PAN) images under the pansharpening framework. Most decimated multi-resolution pansharpening approaches upsample the low-resolution MS image to match the resolution of the PAN image. Consequently, a multi-level wavelet decomposition is performed, where the edge information from the PAN image is injected in the MS image. In this paper, the authors propose a pansharpening framework that eliminates the need of upsampling of the MS image, using a B-Spline biorthogonal wavelet decomposition scheme. The proposed method features similar performance to the state-of-the-art pansharpening methods without the extra computational cost induced by upsampling.
\end{abstract}

\section{INTRODUCTION}

Image Fusion is the process of combining visual information, obtained from various image modalities, into a single image representation, in order to facilitate information inference by human operators or computer vision systems [1]. The launch of several satellites, that provide various types of spectral and spatial information about our planet, have encouraged the development of remote sensing systems. The IKONOS satellite, launched in 2000, offers Multi-Spectral (MS) imagery at $4 \mathrm{~m}$ and Panchromatic (PAN) imagery at $1 \mathrm{~m}$ resolution [2]. The QuickBird satellite, launched in 2001, collects Panchromatic imagery at $60-70 \mathrm{~cm}$ resolution and Multi-Spectral imagery at 2.4 and $2.8 \mathrm{~m}$ resolutions [3]. Current state-of-the-art image processing and fusion methods have facilitated the efficient processing and enhancement of the available satellite image data.

Pansharpening is the procedure of combining the high spectral information available in Multi-Spectral (MS) images with the high spatial information of the Panchromatic (PAN) image. Although the spatial resolution of MS images in modern satellites has significantly increased, PAN imagery still has finer spatial resolution than MS imagery [4]. In contrast, MS imagery provides better spectral resolution compared to PAN imagery (RGB and Night Infra Red (NIR) channel). Both these features are essential for performing image classification tasks in remote sensing applications. High spectral resolution facilitates the discrimination of land cover types, whereas high spatial resolution facilitates the identification of textures and the accurate extraction of shape and boundaries of the different objects present in the image [4]. Assume an MS image $x_{M S}$ of size $M_{1} \times N_{1} \times 3$ (the NIR channel will not be considered in our study) and a PAN image $x_{P A N}$ of size $M_{2} \times N_{2}$ $\left(M_{2} / M_{1}=N_{2} / N_{1}=4\right)$. The problem of Pansharpening can thus be described as the problem of transferring the spatial resolution of the PAN image and the spectral resolution of the MS image to a composite pansharpened image $x_{P S}$ of size $M_{2} \times N_{2} \times 3$.

The literature in Pansharpening methods is vast, covering a wide variety of methods [4]-[11]. The Gram-Schmidt (GS) Pansharpening methods [9], [10] combine the PAN and MS images using the GS transformation. Principal Component Analysis (PCA)-based pansharpening approaches perform PCA to select a principal image representation between the PAN and the MS images [11]. Wavelet-based Pansharpening is a multi-resolution method featuring enhanced performance [4]-[8]. Undecimated wavelet-based approaches using the à-trous algorithm seem to perform better than decimated approaches [8]. Most decimated pansharpening approaches employ an upsampling preprocessing step in order to tackle the problem of spatial resolution difference between the PAN and MS images. In this paper, the authors revise current decimated wavelet-based pansharpening approaches, by proposing a methodology to solve the spatial resolution problem inside the wavelet multi-resolution analysis. The computational cost of a wavelet-based pansharpening approach is thus reduced, while retaining similar image quality.

\section{WAVELET-BASED PANSHARPENING}

Most pansharpening approaches tackle the difference in spatial resolution between the PAN and the MS image by upsampling the three channels (RGB) of the MS image by a factor of 4 in order to match the resolution of the PAN image [4]-[8]. Thus, the first step in most pansharpening systems is upsampling, which is usually performed by Bicubic or B-spline interpolation.

Most fusion approaches create a single channel output from multiple modality inputs, whereas in pansharpening a multichannel fused output is required. A first strategy is to fuse each RGB channel independently with the PAN image to create a RGB Pansharpened image [5]. Another widely used approach is to transfer the RGB MS image to a representation 


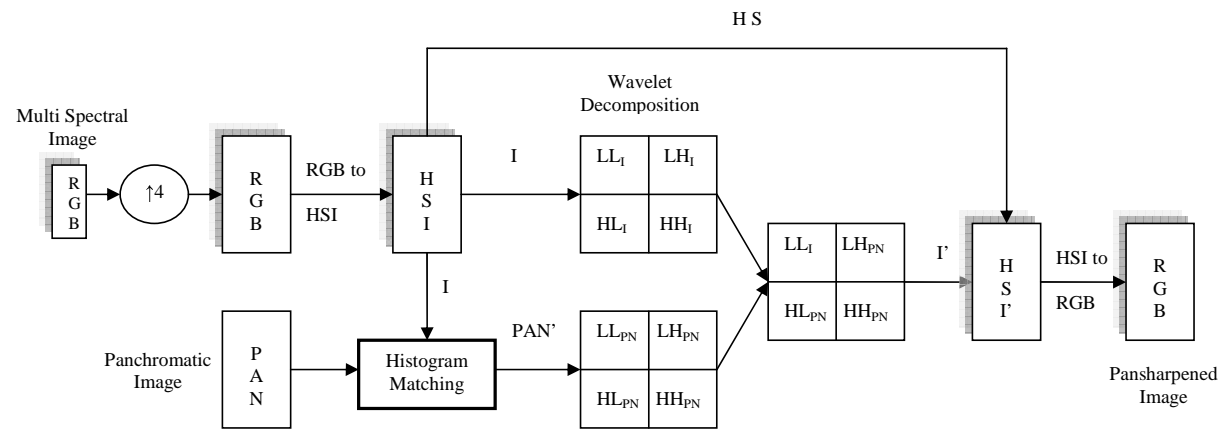

Fig. 1. A common wavelet-based Pansharpening architecture using upsampling to match the different resolution inputs.

of Intensity, Hue and Saturation (IHS) [5]. The intensity image in the IHS representation is very similar to a panchromatic image, thus many approaches fuse the I channel of the MS image with the PAN image and then transform the modified I channel jointly with the original $\mathrm{H}, \mathrm{S}$ channels back to the RGB domain to create the Pansharpened image. There are various implementations of the forward and inverse transformation between the RGB and the IHS colour systems [1], [5]. In this work, we followed the implementation in [12].

The Wavelet Transformation (WT) is a multi-resolution decomposition, where the original image is initially substituted by a structure of approximation images $L L^{j}$ and three detail images $H L^{j}, L H^{j}, H H^{j}$ of lower resolution (usually downsampled by 2) at various decomposition levels $j$. The detail images $H L, L H, H H$ capture the image's salient features, whereas the approximation images $L L$ capture the low frequency information, mainly intensity information.

Based on the previous observation, most wavelet-based pansharpening approaches [4]-[8] perform a $j$-level wavelet decomposition to both the PAN and MS image. Some approaches decompose the channels RGB independently and perform channel pansharpening with the PAN image. Several other approaches transform the MS image to the IHS space and perform wavelet decomposition on the I channel alone. Once the wavelet decompositions are calculated, the wavelet decomposition of the pansharpened image is formed from the calculated decompositions. The detail images $H L^{i}, L H^{i}, H H^{i}, \forall i \in[1, j]$ of the pansharpened image's decomposition are acquired from the PAN image's decomposition. The $L L$ image is acquired from the last $L L^{1}$ of MS image's decomposition. The intensity range of the PAN image is matched to the intensity range of the I channel of the MS image prior to the wavelet decomposition. The intensity matching can be either a simple linear transformation or a nonlinear exact histogram matching, as described in [13]. The pansharpened decomposition is finally recomposed, producing the final pansharpened image (see Fig. 1). In practice, the choice of wavelet family does not seem to affect significantly the performance of pansharpening, although the choice of an undecimated wavelet decomposition (the à-trous algorithm) seems to produce superior results compared to the decimated case [8].

\section{Decimated wavelet-Based Pansharpening WITHOUT UPSAMPLING}

The current wavelet-based schemes feature the following conceptual redundancy. The algorithm is required to process two input images at different resolutions. The authors believe that the upsampling of the MS image can be avoided, since the whole procedure actually implies moving up and down the MS image's scale of decomposition. Instead, one can only move downwards the wavelet decomposition in order to match the resolution of the PAN image and perform pansharpening.

To avoid the upsampling stage of the MS image, the different resolutions are matched inside the wavelet decomposition. Initially, we move the MS image to the IHS space and store the H,S components. Then, wavelet decomposition of $j-2$ levels of the I image is performed. Wavelet decomposition of $j$ levels is performed to the PAN image and the same pansharpening procedure can now be applied in a similar manner to the previous wavelet-based approaches. The $L L^{1}$ is acquired from the decomposition of the I image, whereas the $H L^{i}, L H^{i}, H H^{i}, \forall i \in[1, j]$ images are acquired from the decomposition of the PAN image. Hence, a coefficient substitution method is followed rather than coefficient fusion to increase computational complexity. Once the PS image is formed in the wavelet domain, wavelet recomposition is used to reconstruct the I image to the resolution of the PAN image (up $j$ levels). The $\mathrm{H}$ and $\mathrm{S}$ channels can be upsampled using a two-level wavelet recomposition, by placing them as the LL sub-image and filling the $H L, L H, H H$ sub-images with zeros, or using bicubic interpolation. Finally, the pansharpened image $x_{P S}$ is acquired by returning the estimated IHS representation to the RGB space. The whole procedure is outlined in Figure 2.

In order to combine the I channel of the MS image with the PAN image in the wavelet decomposition, one has to match the value range of the PAN image to the value range of the I channel, regardless of the difference in resolution. Many approaches employ an exact histogram specification approach, as proposed by Coltuc et al [13]. This approach often introduced 


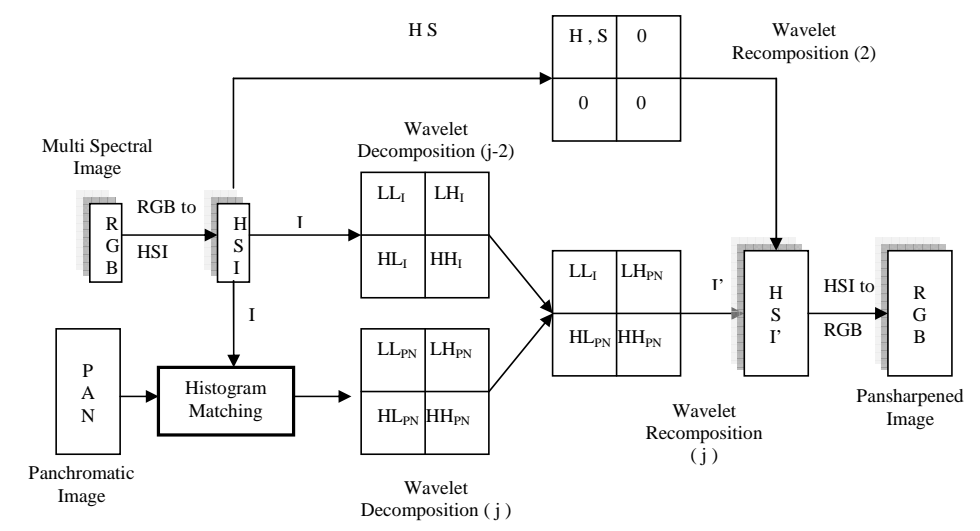

Fig. 2. The proposed wavelet-based Pansharpening architecture that involves no upsampling of the multispectral input. The number in brackets denotes the level of wavelet decomposition/recomposition.

artifacts in our pansharpening examples, thus, we employed a linear mapping in the form of $\tilde{x}_{P A N}=a x_{P A N}+b$, where $a, b$ are estimated to impose the mean and variance of the I channel of the MS image on $\tilde{x}_{P A N}$. This process yields a smoother histogram matching procedure with less artifacts than the nonlinear specification approach in [13].

One can argue that the proposed approach will suffer from edge localisation problems. These problems will occur due to the difference in the number of wavelet decomposition levels that are performed on the two images. The wavelet decomposition may introduce some shifting in the actual position of the edges as it moves to the coarsest decomposition level. Thus, using a $(j-2)$-level decomposition for the MS image and a $j$-level decomposition for the PAN image may result in a slight drift between the edges in the two representations and thus double edges will appear in the pansharpened image after reconstruction. This problem does not appear in the original wavelet-based methods, since the same level of wavelet decomposition is applied on both input images and possible correction to edge localisation is performed via registration after upsampling. In the proposed scheme this problem can be alleviated by using a wavelet family with strong edge localisation properties, so that that the wavelet decomposition introduces minimal shift to the original position of the edges. The Biorthogonal Spline Wavelets are shown to provide accurate edge localisation along the wavelet decomposition, as they provide optimal spatial-frequency localisation [14]. In this analysis, the Toolbox Wavelets' implementation of Biorthogonal Spline Wavelets (Cohen-Daubechies-Feauveau CDF) $(3,9)$ is employed to perform the wavelet decomposition [15]. Using the aforementioned wavelet family, one can encounter minimal edge localisation problems in the pansharpened image, without the extra registration and upsampling stages.

In this paper, we examine the case that the input images are registered or have minimal registration offset. In the case of strong misregistration between the MS and the PAN images, one can register the MS channels with the $L L$ subimage of the two-level decimated decomposition of the PAN image. Upsampling an image does not necessarily improve the localisation of edges in the MS channels and thus performing registration at a level with increased uncertainty in the edge position does not necessarily improve the registration and pansharpening performance.

\section{Computational Cost Comparison}

In order to compare the computational cost of the two wavelet-based methods, we will assume that the upsampling of the H,S channel in the proposed method is performed by bicubic interpolation. Thus, the difference in the computational cost between the two methods is entailed in the following steps. In the original wavelet-based framework, a single channel bicubic upsampling by a factor of 4 is performed, an RGB to IHS transformation at resolution $M_{2} \times N_{2}$ and a wavelet decomposition of $j$ levels. In the proposed framework, these steps are replaced by an RGB to IHS transformation at resolution $M_{1} \times N_{1}\left(M_{1}<M_{2}, N_{1}<N_{2}\right)$ and a wavelet decomposition of $j-2$ levels. Hence, the computational cost is reduced in the proposed framework.

\section{EXPERIMENTS}

In this section, the performance and the computation time of three wavelet-based pansharpening schemes along with a PCA-based method are evaluated. The "Toolbox Wavelet" implementation of Biorthogonal Spline Wavelets (CohenDaubechies-Feauveau CDF) $(3,9)$ and the ' a-trous transformation was employed [15]. Four levels of wavelet decomposition $(j=4)$ were performed. A recent study has shown that this is the optimal level of wavelet decomposition for pansharpening [16]. Pansharpening performance was evaluated in terms of Relative Shift, Deviation Index, Spectral Angle, Correlation Index, ERGAS and $Q_{4}$, as commonly employed by the Pansharpening community [1], [5], [7]. The computation time was evaluated using MATLAB's "tic"-"toc" commands on a Core $23 \mathrm{GHz}$ PC with $4 \mathrm{~GB}$ RAM.

Experiments employed three QuickBird image pairs of different terrain types, available online from the University 


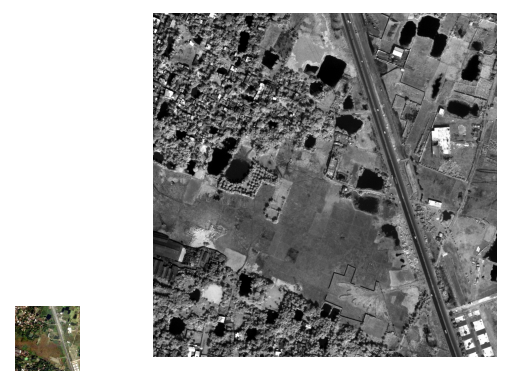
(a) MS
(b) PAN Sensor
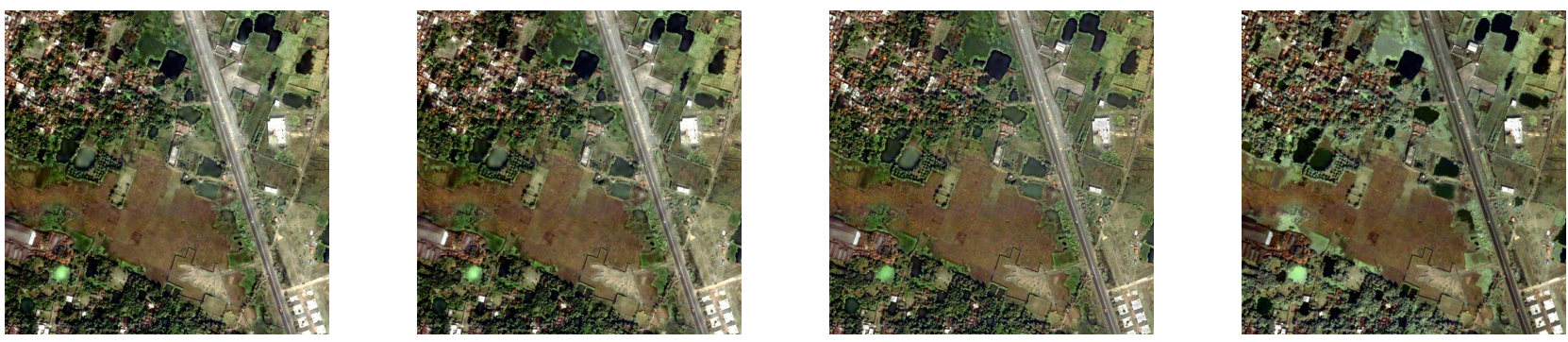

(c) Decimated WT - No Upsampling (d) Decimated WT - With Upsam-

(e) À-trous WT - With Upsampling pling

Fig. 3. Comparison of four Pansharpening schemes using a sample from the QuickBird database.

(f) PCA-based
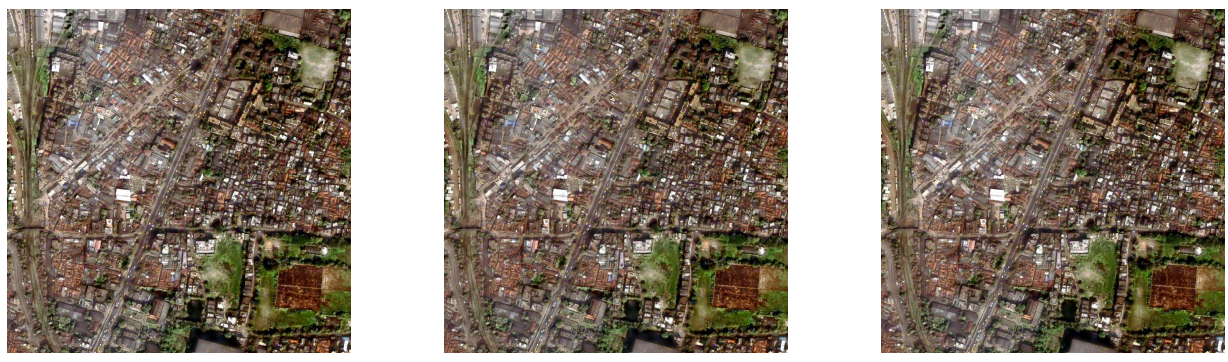

(c) Decimated WT - No Upsampling (d) Decimated WT - With Upsam- (e) À-trous WT - With Upsampling pling

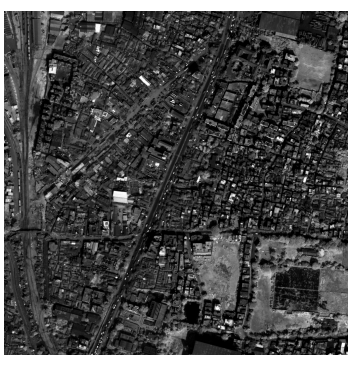

(b) PAN Sensor (a) MS

Sensor

Fig. 4. Comparison of four Pansharpening schemes using a sample from the QuickBird database.

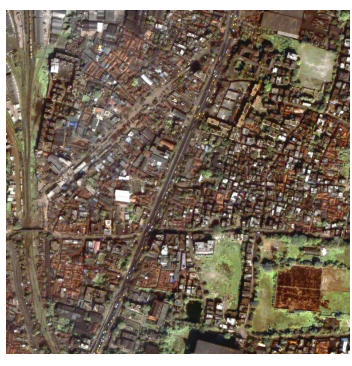

(f) PCA-based 
of Maryland ${ }^{1}$, that were used to construct 256, 60 and 120 $1024 \times 1024$ PAN and $256 \times 256$ MS images respectively from the three image pairs. The images were already orthorectified. We applied the three wavelet-based approaches and a PCA-based approach that employs upsampling. We performed numerical evaluation of the achieved pansharpening with reference to the original MS image for those metrics that measure spectral deviation and with original PAN image for those metrics that measure spatial deviation. Average results for each performance index are summarised in Table I. It should be noted that wavelet-based methods are superior to PCA-based methods. It is clear that the undecimated à-trous waveletbased pansharpening excels in all metrics, as it was expected. However, the wavelet-based approach without upsampling seems to be similar in performance to the original approach, which implies that there was no reduction in performance removing the upsampling stage. In Fig.3, 4, 5 we can perform a visual evaluation of the aforementioned approaches.

One important difference is the computation time between the wavelet-based approaches, which is also depicted in Table I. The decimated approach without upsampling required $4.28 \mathrm{sec} / \mathrm{image}$ on average to perform pansharpening on the aforementioned machine, whereas the approach with upsampling required $6.09 \mathrm{sec} / \mathrm{image}$ to perform the same task with almost similar quality. The à-trous wavelet approach required 9.85 sec/image. This implies that the proposed scheme has reduced the processing time by $\sim 30 \%$ compared to the decimated wavelet transform, which is quite significant for real-time applications. The PCA method was obviously the fastest method ( $0.96 \mathrm{sec} / \mathrm{image})$ in all experiments as fusion in essentially performed in the spatial domain without any multiscale decomposition. However, the PCA method featured the worst pansharpening performance. Finally, there was no visible registration error in any of the images for the waveletbased approach without upsampling. This was achieved by using Biorthogonal B-spline wavelets with accurate edge localisation properties.

\section{CONCLUSIONS}

In this paper, the authors reviewed the current state-ofthe-art wavelet-based methods and identified the redundant step of upsampling the multispectral image to the resolution of the panchromatic image in order to perform a wavelet decomposition for image fusion. Instead, we proposed to remove the upsampling stage and perform different-level wavelet decompositions in order to match the resolution of the two images and perform fusion of the spectral and spatial information. Experimental results on different terrain types show similar performance to decimated wavelet-based methods with upsampling, but with decreased computational complexity.

\section{REFERENCES}

[1] T. Stathaki, Image Fusion: Algorithms and Applications, Academic Press, 2008.

\footnotetext{
${ }^{1}$ http://glcf.umiacs.umd.edu/data/quickbird/index.shtml
}

TABLE I

Average Pansharpening Performance using Relative Shift, DeViation Index, Spectral Angle, Correlation IndeX, ERGAS, $Q_{4}$ FOR THE EXAMINED SCHEMES.

\begin{tabular}{|c|c|c|c|c|}
\hline Index & $\begin{array}{c}\text { Wavelet } \\
\text { Decimated } \\
\text { NoUpsample }\end{array}$ & $\begin{array}{c}\text { Wavelet } \\
\text { Decimated } \\
\text { Upsample }\end{array}$ & $\begin{array}{l}\text { Wavelet } \\
\text { à-trous } \\
\text { Upsample }\end{array}$ & PCA \\
\hline \multicolumn{5}{|c|}{ Set 1} \\
\hline $\begin{array}{c}\text { Relative } \\
\text { Shift }\end{array}$ & 7.68 & 9.34 & 7.14 & 28.43 \\
\hline $\begin{array}{l}\text { Deviation } \\
\text { Index }\end{array}$ & 1.29 & 1.38 & 1.15 & 12.33 \\
\hline $\begin{array}{c}\text { Spectral } \\
\text { Angle }\end{array}$ & 3.45 & 3.35 & 3.2 & 3.95 \\
\hline $\begin{array}{l}\text { Correla- } \\
\text { tion Index }\end{array}$ & 0.844 & 0.842 & 0.861 & 0.532 \\
\hline ERGAS & 6.703 & 6.535 & 6.16 & 10.19 \\
\hline$Q_{4}$ & 0.684 & 0.687 & 0.712 & 0.554 \\
\hline $\begin{array}{l}\text { CPU time } \\
\text { /frame }(\mathrm{sec})\end{array}$ & 4.23 & 6.04 & 9.73 & 0.94 \\
\hline \multicolumn{5}{|c|}{ Set 2} \\
\hline $\begin{array}{c}\text { Relative } \\
\text { Shift }\end{array}$ & 43.18 & 51.87 & 41.07 & 86.87 \\
\hline $\begin{array}{l}\text { Deviation } \\
\text { Index }\end{array}$ & 7.45 & 8.62 & 6.48 & 22.79 \\
\hline $\begin{array}{l}\text { Spectral } \\
\text { Angle }\end{array}$ & 5.89 & 5.85 & 5.58 & 6.3 \\
\hline $\begin{array}{l}\text { Correla- } \\
\text { tion Index }\end{array}$ & 0.83 & 0.83 & 0.85 & 0.676 \\
\hline ERGAS & 9.23 & 9.03 & 8.51 & 10.99 \\
\hline$Q_{4}$ & 0.74 & 0.74 & 0.76 & 0.69 \\
\hline $\begin{array}{l}\text { CPU time } \\
\text { /frame (sec) }\end{array}$ & 4.38 & 6.17 & 9.49 & 0.95 \\
\hline \multicolumn{5}{|c|}{ Set 3} \\
\hline $\begin{array}{c}\text { Relative } \\
\text { Shift }\end{array}$ & 9.88 & 11.24 & 7.22 & 43.77 \\
\hline $\begin{array}{l}\text { Deviation } \\
\text { Index }\end{array}$ & 1.34 & 1.48 & 1.64 & 16.09 \\
\hline $\begin{array}{l}\text { Spectral } \\
\text { Angle }\end{array}$ & 2.94 & 2.97 & 2.67 & 5.22 \\
\hline $\begin{array}{l}\text { Correla- } \\
\text { tion Index }\end{array}$ & 0.76 & 0.75 & 0.8 & 0.2 \\
\hline ERGAS & 5.8 & 5.67 & 5.36 & 9.97 \\
\hline$Q_{4}$ & 0.58 & 0.58 & 0.61 & 0.62 \\
\hline $\begin{array}{l}\text { CPU time } \\
\text { /frame (sec) }\end{array}$ & 4.25 & 6.077 & 10.34 & 1.01 \\
\hline
\end{tabular}

[2] Wikipedia, "IKONOS satellite - http://en.wikipedia.org/wiki/IKONOS,"

[3] Wikipedia, "QuickBird satellite-http://en.wikipedia.org/wiki/Quickbird,"

[4] M.M. Khan, J. Chanussot, L. Condat, and A. Montanvert, "Indusion: Fusion of multispectral and panchromatic images using the induction scaling technique," IEEE Geoscience and Remote Sensing Letters, vol. 5, no. 1, pp. 98-102, 2008.

[5] Y. Zhang and G. Hong, "An IHS and wavelet integrated approach to improve pan-sharpening visual quality of natural colour IKONOS and QuickBird images," Elsevier Information Fusion, vol. 6, no. 3, pp. 225234,2005

[6] V.P. Shah, N.H. Younan, and R. King, "Pan-sharpening via the contourlet transform," in Proc. IEEE Int. Conf. on Geoscience and Remote Sensing Symposium (IGARSS), 2007, pp. 310 - 313.

[7] L. Alparone, L. Wald, J. Chanussot, C. Thomas, P. Gamba, and L.M. Bruce, "Comparison of pansharpening algorithms: Outcome of the 2006 GRS-S data-fusion contest," IEEE Transactions on Geoscience and Remote Sensing, vol. 45, no. 10, pp. 3012 - 3021, 2007.

[8] B. Aiazzi, L. Alparone, S. Baronti, and A. Garzelli, "Context-driven fusion of high spatial and spectral resolution images based on oversampled multiresolution analysis," IEEE Transactions on Geoscience and 


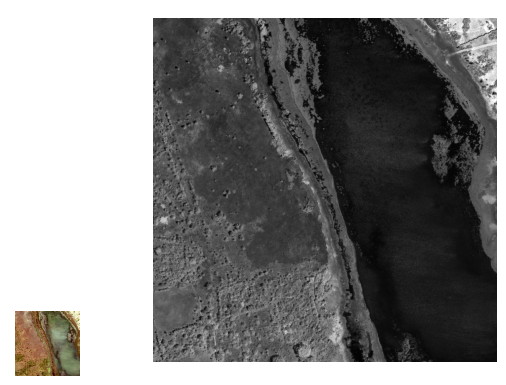

(a) MS

(b) PAN Sensor

Sensor
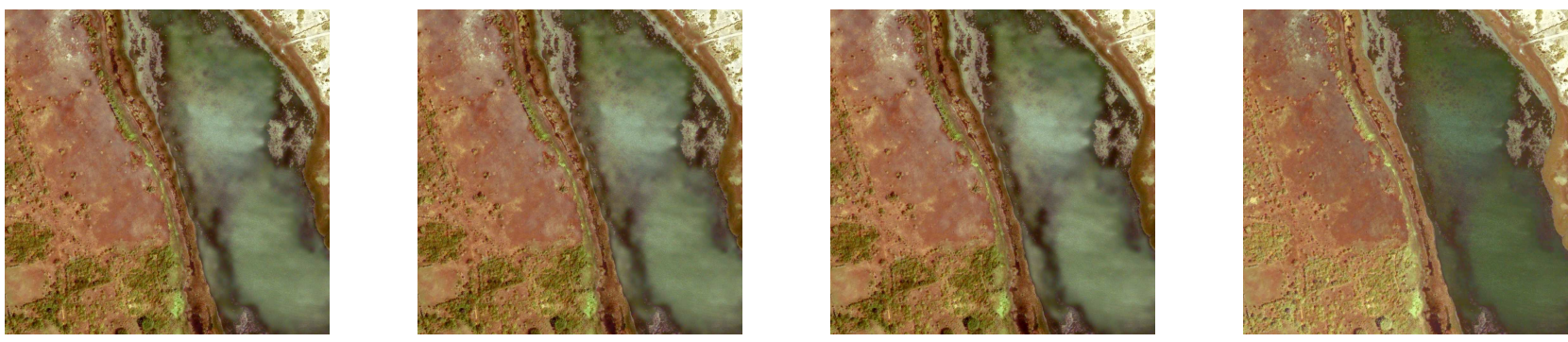

(c) Decimated WT - No Upsampling

(d) Decimated WT - With Upsam-

(e) À-trous WT - With Upsampling

(f) PCA-based

Fig. 5. Comparison of four Pansharpening schemes using a sample from the QuickBird database.

Remote Sensing, vol. 40, no. 10, pp. 2300- 2312, 2002.

[9] B. Aiazzi, S. Baronti, and M. Selva, "Improving component substitution pansharpening through multivariate regression of MS+PAN data," IEEE Transactions on Geoscience and Remote Sensing, vol. 45, no. 10, pp. 98-102, 2007.

[10] C.A. Laben and B.V. Brower, "Process for enhancing the spatial resolution of multispectral imagery using pan-sharpening," Tech. Rep., Eastman Kodak Company, 2000.

[11] V.K. Shettigara, "A generalized component substitution technique for spatial enhancement of multispectral imagesusing a higher resolution data set," Photogramm Eng. Remote Sens., vol. 58, no. 5, pp. 561-567, 1992.

[12] P. Getreuer, "Colorspace MATLAB function - converting between color spaces, available from http://www.math.ucla.edu/ getreuer/colorspace.html," .

[13] D. Coltuc, P. Bolon, and J.-M. Chassery, "Exact histogram specification," IEEE Transactions on Image Processing, vol. 15, no. 5, pp. 1143 - 1152, 2006.

[14] M. Unser, "Splines: A perfect fit for signal and image processing," IEEE Signal Processing Magazine, vol. 16, no. 6, pp. 22-38, 1999.

[15] Gabriel Peyre, "Toolbox wavelets - http://www.ceremade.dauphine.fr/ $\sim$ peyre/matlab/wavelets/content.html," 2009.

[16] P.S. Pradhan, R.L. King, N.H. Younan, and D.W. Holcomb, "Estimation of the number of decomposition levels for a wavelet-based multiresolution multisensor image fusion," IEEE Transactions on Geoscience and Remote Sensing, vol. 44, no. 12, pp. 3674 - 3686, 2006. 